\title{
ANÁLISIS DE LA REGENERACIÓN NATURAL DE LA VEGETACIÓN EN TALUDES DE CAMINOS DE LA RUTA Y-85, PARQUE KARUKINKA, TIERRA DEL FUEGO, CHILE
}

\author{
ANALYSIS OF NATURAL REGENERATION OF VEGETATION ON ROAD SLOPES \\ OF RUTA Y-85, KARUKINKA PARK, TIERRA DEL FUEGO, CHILE
}

Fiorella Repetto-Giavelli ${ }^{1} \&$ Ernesto Teneb B. ${ }^{2}$

\begin{abstract}
In this study we analyzed the pattern of vegetal succession of 38 road slopes formed by the construction of the Route Y-85, which connects Estancia Vicuña with Yendegaia (54 20 'S, 68 $45^{\circ}$ ' W) in Chilean Tierra del Fuego. Our results indicate that both coverage and species richness show significant differences between the degraded road slopes and the reference sites in the four studied ecosystems. Most of the species that established themselves on degraded road slopes came from the reference ecosystems, the most abundant species were Acaena magellanica, Deschampsia flexuosa and Nothofagus pumilio. The road slopes with greater vegetation cover of species were found in the forest ecosystem (less than $25 \%)$, followed by ecotone ecosystem. These results suggest that the natural restoration of road slopes in southern Tierra del Fuego is an extremely slow process, making it necessary to start an "active" restoration process.
\end{abstract}

Key words: Road slope, Passive restoration, Succession, Karukinka Park, Tierra del Fuego, Patagonia.

\section{RESUMEN}

En este estudio analizamos el patrón de sucesión vegetal de 38 taludes de camino formados durante la construcción de la ruta Y-85, que une Estancia Vicuña con Yendegaia (54 20’ S; 68 45’ W), en Tierra del Fuego chilena. Nuestros resultados indican que en los cuatro ecosistemas estudiados, tanto la cobertura como la riqueza de especies, presentan diferencias significativas entre los taludes degradados y el sitio de referencia respectivo. La mayoría de las especies que lograron establecerse en taludes degradados provienen de los ecosistemas de referencia, siendo las más abundantes Acaena magellanica,

$1 \quad$ Wildlife Conservation Society - Chile. frepetto@wcs.org

2 Grupo de Estudios Ambientales, Universidad de Magallanes, Punta Arenas, Chile. 
Deschampsia flexuosa y Nothofagus pumilio. Los taludes con mayor cobertura vegetal se encuentran en el ecosistema de bosque (menores al 25\%) seguido por el ecosistema de ecotono. Sugiriendo que la restauración natural de los taludes en el sur de Tierra del Fuego es un proceso extremadamente lento, haciéndose necesario iniciar procesos de restauración "activa".

Palabras Clave: Talud, Restauración pasiva, Sucesión, Parque Karukinka, Tierra del Fuego, Patagonia.

\section{INTRODUCCIÓN}

La construcción de infraestructura vial es uno de los instrumentos más utilizados en las políticas de desarrollo local, estrategia que intenta aportar tanto al progreso social como al desarrollo sostenible (Vásquez-Barquero, 2009). La construcción de caminos, conlleva múltiples beneficios a la sociedad, destacando: la integración del patrimonio, oportunidades y mejoras de comercio, desarrollo rural/ urbano y comunicación. Asimismo, permite acceder a una serie de servicios ecosistémicos no tangibles: recreacionales, estéticos, educativos, y el realce de la belleza escénica (Jackson \& Hobbs, 2009). Sin embargo, también se producen efectos negativos sobre el patrimonio natural, como disminución de la calidad de las aguas, del aire, pérdida de hábitat para la vida silvestre, fragmentación y degradación del paisaje y de la vegetación circundante (Forman et al. 2003, National Research Council 2005). El impacto negativo del desarrollo de caminos puede, sin embargo, ser minimizado considerando explícitamente, desde el inicio del proyecto vial, parámetros ambientales e incorporándolos al diseño del mismo (Gómez-Orea \& Gómez-Villarino, 2007). De manera alternativa (o incluso complementaria) los efectos de este impacto pueden ser mitigados incorporando medidas de restauración de los caminos una vez terminadas las faenas iniciales de construcción, favoreciendo el re-establecimiento de la integridad biótica existente de forma previa a la intervención, establecida en términos de composición de especies, estructura comunitaria, procesos ecosistémicos, productividad y/o belleza escénica (Jackson \& Hobbs, 2009).

Las plantas tienen un rol importante en los ecosistemas, al proteger el suelo de la erosión y la escorrentía, además de permitir la acumulación de partículas finas y la acumulación de nutrientes en su forma disponible (Bradshaw, 1997), siendo esto aún más importante en áreas degradadas por construcción de caminos al estabilizar taludes y terraplenes (Snelder \& Bryan, 1995, Andrés \& Jorba, 2000), los cuales son construidos por acumulación y compactación de material de un área adyacente, formando áreas con suelos de baja fertilidad, pedregosidad superficial, acusadas pendientes y falta de estructura (Tormo et al. 2006), suelos pobremente consolidados, muy vulnerables a la erosión (Nicolau 2002). En taludes de camino, la baja o nula cobertura vegetal ha sido atribuida principalmente a estas condiciones desfavorables del suelo, las que afectan el establecimiento de algunas plantas, por lo tanto en caso que la disponibilidad de semillas no sea la primera barrera para la colonización exitosa como se ha demostrado (Foster \& Tilman, 2003), el arribo de semillas no es garantía de colonización exitosa en estos sectores (Tormo et al. 2006).

Ciertas especies tendrán mayor éxito, y serán más beneficiosas en etapas tempranas, por ejemplo especies pioneras y de etapas intermedias, capaces de modificar el ambiente, tanto biótico como abiótico, son clave para poner en marcha el proceso sucesional lo que permitirá la recuperación, a largo plazo, de buena parte de la vegetación existente antes de la perturbación (Zamora et al. 2004).

Las especies idóneas a introducir suelen seleccionarse preferentemente entre las existentes en medios próximos no degradados, con el fin de garantizar su adaptación a las condiciones adversas en las que deben establecerse. La selección de especies se basa esencialmente en: a) adaptación a condiciones climáticas, b) elevada cobertura protectora potencial, c) adaptación a perturbaciones (fuego, ramoneo) (Cortina et al. 2004).

El Parque Karukinka, área protegida propiedad de Wildlife Conservation Society (WCS) localizada al sur de la Isla de Tierra del Fuego, tiene una superficie de 300.000 ha (Saavedra et al. 2011), con acceso terrestre gracias a la Ruta Y-85 (Camino Estancia Vicuña-Yendegaia), cruzándola completamente de norte a sur en su extremo oriental. La construcción 
de este camino fue iniciada en 1995 y todavía continúa, pues se pretende alcanzar el Canal Beagle en los próximos años. La construcción de esta ruta ha impactado los diversos ecosistemas que existen en el Parque, y continuará afectando otras áreas a medida que continúe su construcción hacia el sur. El estándar ambiental de esta ruta fue evaluado en un trabajo conjunto de WCS y la Dirección de Vialidad de Magallanes, el cual ha sido ampliamente difundido en la región. Este análisis identificó 7 tipos de riesgos ambientales en la ruta, siendo los taludes el principal riesgo, con el 37\% del total de puntos identificados (Repetto, 2009). Los taludes podrían recuperar su vegetación espontáneamente, a través de procesos como la colonización y sucesión, como se postula que ocurre en diversas áreas perturbadas (Novák \& Prach, 2003). Es por esto que evaluamos la composición florística de taludes y de áreas de referencia en la Ruta Y-85, en el Parque Karukinka, con el objetivo de evaluar la dinámica de regeneración vegetal que se está dando en el lugar, además de identificar y proponer especies útiles para una estrategia de restauración activa en el lugar, en caso de ser necesaria.

\section{MATERIALES Y MÉTODO}

El estudio fue realizado en la porción sur de la isla de Tierra del Fuego-Chile, en el Parque Karukinka (54 $8^{\prime}$ S, $68^{\circ} 42^{\prime}$ W) (Fig. 1), la cual es cruzada de $\mathrm{N}$ a S por la Ruta Y-85 (Camino Estancia Vicuña-Yendegaia), único camino público que alcanza el sur de Tierra del Fuego, Región de Magallanes-Chile (Saavedra et al. 2011).

Este camino se encuentra actualmente en construcción, ampliando la conectividad terrestre en esta zona. Se inicia en la Estancia Vicuña, cercano al sector Vicuña de Karukinka donde se encuentra

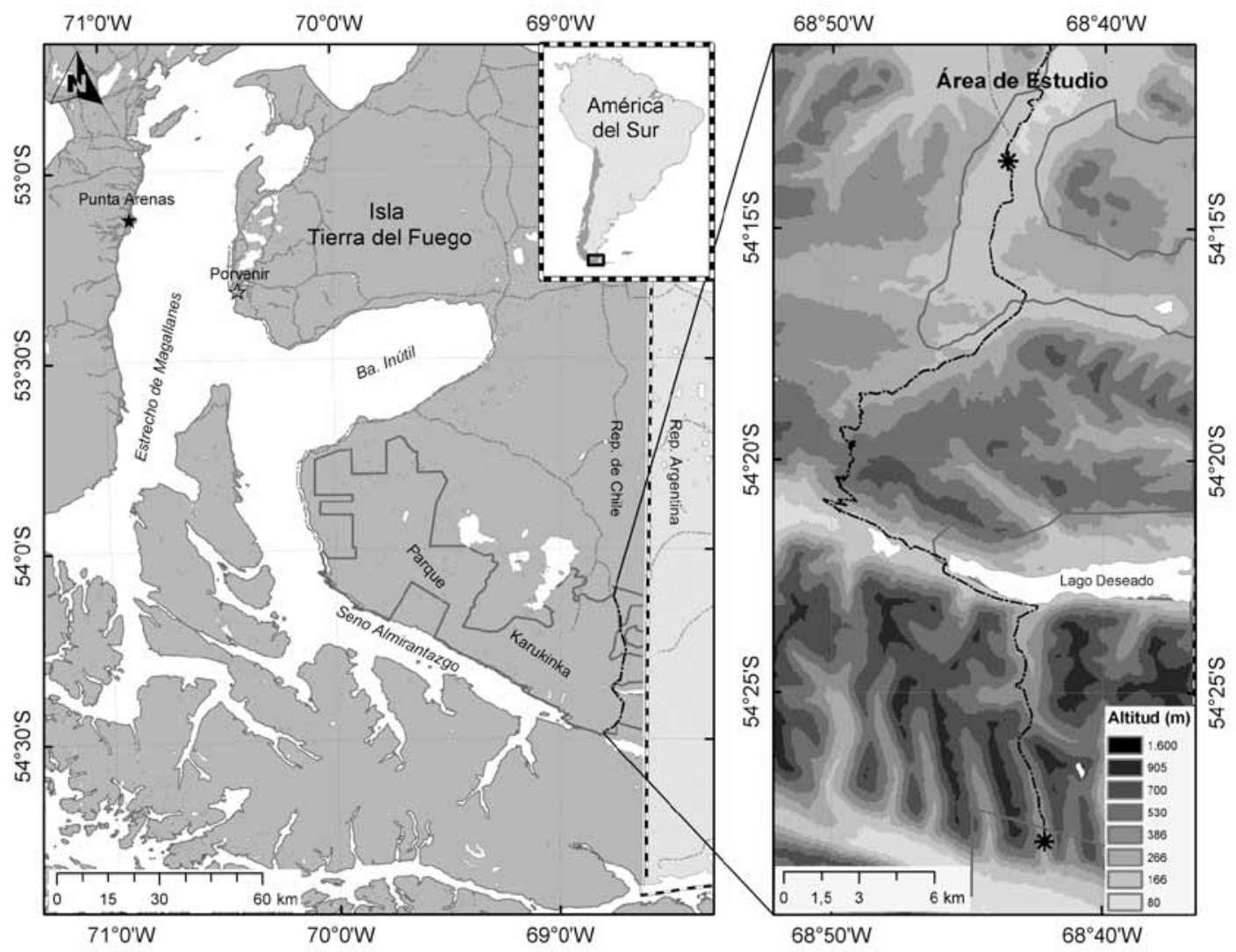

Fig. 1. Mapa del Parque Karukinka señalando el área de estudio, como parte del Camino Estancia Vicuña-Yendegaia (Ruta Y-85). Región de Magallanes, Chile. 
una de las guarderías del Parque, y espera llegar al Canal Beagle en un futuro cercano. En su trayecto atraviesa diversas geoformas, incluyendo Lago Deseado, Valle del Río Sánchez, Lago Fagnano, entre otros.

Los datos obtenidos en este estudio corresponden al tramo de la Ruta Y-85 que comienza en el Puente Río Rasmussen ( $\mathrm{km} \mathrm{0)} \mathrm{y} \mathrm{termina} \mathrm{en} \mathrm{el}$ kilómetro 51, en las cercanías del Lago Fagnano, contenido casi en su totalidad dentro del Parque Karukinka (Repetto, 2009). Su construcción estuvo a cargo del Cuerpo Militar del Trabajo (CMT), comenzó el año 1995 y finalizó el año 2007 (Fig. 1).

Durante el verano austral (enero-febrero) del año 2009, fueron evaluados 38 sitios de muestreo distribuidos en 4 tipos de ecosistemas: Estepa (E), Bosque Caducifolio (B), el Ecotono entre ambos (T) y Estepa Andina (VA). En cada sitio se ubicaron dos parcelas de $10 \times 15 \mathrm{~m}$, en cada parcela se dispuso al azar entre 6 y 8 cuadrantes de $0,5 \times 0,5 \mathrm{~m}$ de lado. En cada cuadrante se midió la cobertura de la vegetación individualizando las especies presentes.

En cada sitio, se dispuso una parcela en el talud o corte formado por la construcción del camino, correspondiente al área degradada (identificada con el número 2); en tanto que la segunda parcela se dispuso por encima del corte del talud, correspondiente al área de referencia donde se ubicaron las parcelas control (identificadas con el número 1). Del total de 38 sitios de muestreo, se ubicaron 10 en cada ecosistema de Estepa (E), Ecotono Estepa-Bosque (T) y Bosque Caducifolio (B), y 8 sitios en Estepa Andina (VA). A su vez, los sitios fueron diferenciados según tipo de sustrato observado en el corte, siendo la mitad del total de sitios de Corte en Roca (CR) y la otra mitad de Terreno de Cualquier Naturaleza (TCN) donde predominan los limos y arcillas, algunas veces mezclados con gravas de distinto tipo. Los sitios de muestreo fueron georreferenciados con un navegador GPS, utilizando para ello el sistema UTM.

Los datos de riqueza y cobertura de especies de cada parcela fueron tabulados, creando una matriz recíproca con la información para el área degradada y su área de referencia respectiva. Con esta información se aplicaron índices de distancia de Bray-Curtis y análisis de componentes principales (PCA) con el fin de establecer las tendencias y patrones de la flora y vegetación en las áreas degradadas. En particular y con fines estadísticos, los datos de cobertura de especies expresado como porcentaje de recubrimiento fueron transformados a arco-seno o transformación angular, con el fin de cumplir con la homocedasticidad y el ajuste normal de la distribución de los datos (Sokal \& Rohlf, 1995, Bonham 1989), ello con el fin de aplicar un análisis de varianza (ANOVA) y test de Tukey que permita establecer la comparaciones estadísticas entre: tipos de talud y tipos de ecosistema. Asimismo, los datos de riqueza de especies de las áreas degradada y de referencia fueron comparadas con el estadístico no paramétrico U Mann-Whitney. Los análisis estadísticos fueron realizados en los software SPSS Inc y MVSP.

Las plantas observadas fueron identificadas en terreno hasta el nivel de especie, utilizando el apoyo de claves taxonómicas presente en la literatura especializada.

\section{RESULTADOS}

Fueron identificadas un total de 42 taxa de plantas vasculares, una especie de musgo además de otras criptógamas (Tabla 1), el ecosistema con mayor riqueza de especies en este estudio fue el bosque caducifolio con 31 plantas vasculares, seguido por la estepa (26) y el ecotono (26). El análisis florístico representado por el dendrograma de distancia de Bray-Curtis (Fig. 2) separa los sitios de muestreo en cuatro grupos a una distancia mínima de 63\%. El primer clado, segregado en una distancia mínima de 53,4\% (clado A), está conformado por parcelas del área de referencia de la estepa, ecotono y estepa andina; las especies que la identifican son: Deschampsia flexuosa (L) Trin., Acaena magellanica (Lam.) Vahl, Berberis microphylla G. Forst., Blechnum penna-marina (Poir.) Kuhn, Bolax gummifera (Lam.) Spreng., Gaultheria pumila (L.f.) D.J. Middleton y Rubus geoides $\mathrm{Sm}$. El clado B, reunido bajo una distancia mínima de 54,8\%, está conformado por parcelas pertenecientes al área de bosque caducifolio, incorporándose en este clado una parcela del ecotono; las especies que la caracterizan son: A. magellanica, Phleum alpinum L., Gamochaeta spiciformis (Sch.Bip.) Cabrera, Gunnera magellanica Lam., Nothofagus pumilio (Poepp. \& Endl.) Krasser y el musgo Sphagnum magellanicum Brid. El clado $\mathrm{C}$ conformado por dos parcelas de áreas degradadas, una de la estepa y una de la estepa andina, reunidas a una distancia mínima de 34,9\% 
Tabla 1. Riqueza de especies y cobertura promedio (\%) de las plantas encontradas en los taludes estudiados del parque Karukinka, Tierra del Fuego. 1 = Talud referencia, 2 = Talud degradado, $\mathrm{CR}=$ Corte en roca, TCN = Terreno de cualquier naturaleza. Más detalles en Materiales y métodos. * Criptógamas.

\begin{tabular}{|c|c|c|c|c|c|c|c|c|c|c|c|c|c|c|c|c|}
\hline \multirow{3}{*}{$\begin{array}{l}\text { Ecosistema } \\
\text { Tipo de Corte } \\
\text { Especie / Talud }\end{array}$} & \multicolumn{4}{|c|}{ Estepa } & \multicolumn{4}{|c|}{$\begin{array}{c}\text { Ecotono } \\
\text { estepa-bosque }\end{array}$} & \multicolumn{4}{|c|}{ Bosque caducifolio } & \multicolumn{4}{|c|}{ Vegetación andina } \\
\hline & \multicolumn{2}{|c|}{$\mathrm{CR}$} & \multicolumn{2}{|c|}{$\mathrm{TCN}$} & \multicolumn{2}{|c|}{$\mathrm{CR}$} & \multicolumn{2}{|c|}{$\mathrm{TCN}$} & \multicolumn{2}{|c|}{$\mathrm{CR}$} & \multicolumn{2}{|c|}{$\mathrm{TCN}$} & \multicolumn{2}{|c|}{$\mathrm{CR}$} & \multicolumn{2}{|c|}{$\mathrm{TCN}$} \\
\hline & 1 & 2 & 1 & 2 & 1 & 2 & 1 & 2 & 1 & 2 & 1 & 2 & 1 & 2 & 1 & 2 \\
\hline Acaena magellanica (Lam.) Vahl & 11,7 & & 42,5 & 71,0 & 17,0 & 61,0 & 84,6 & 8,0 & 23,5 & 35,0 & 25,4 & 15,3 & 17,0 & & 16,2 & \\
\hline Berberis microphylla G. Forster & 63,3 & & 11,4 & 13,0 & & & 17,3 & & 77,5 & & 88,0 & & 58,5 & & 53,0 & \\
\hline Blechnum penna-marina (Poir.) Kuhn & 28,3 & & 59,0 & & 54,8 & & 69,0 & & & & 33,0 & & 83,0 & & 13,8 & \\
\hline Bolax gummifera (Lam.) Spreng. & 93,0 & & 15,2 & 13,0 & & & 11,0 & & 2,0 & & & & 13,7 & & 12,0 & \\
\hline Caltha sagittata Cav. & & & & & & & & & & & & & 42,0 & & 25,0 & \\
\hline Cardamine glacialis (G. Forster) DC. & & & & & & & & & & & 13,1 & 17,0 & & & 12,0 & 18,0 \\
\hline Cerastium arvense $\mathrm{L}$. & & & 21,0 & & 1,0 & & & & & & & & & & & \\
\hline Cerastium fontanum Baumg. & & & & & & & & & & & & & & & & \\
\hline Chiliotrichum diffusum (G. Forst.) Kuntze & & & 41,5 & & 8,0 & & & & 7,0 & & & & & & & \\
\hline Deschampsia flexuosa (L.) Trin. & 17,6 & 35,5 & 2,0 & 42,3 & 41,0 & & 5,0 & 35,7 & 5,0 & & & & 58,0 & & 12,5 & \\
\hline Dysopsis glechomoides (A. Rich.) Müll. Arg. & & & & & 41,5 & & 18,3 & & & & 4,0 & & & & & \\
\hline Drapetes muscosus Banks ex Lam. & & & & & & & & & & & & & 38,7 & & 5,0 & \\
\hline Empetrum rubrum Vahl ex Willd. & & & 71,0 & & 3,0 & & & & 5,0 & & & & 27,5 & & & \\
\hline $\begin{array}{l}\text { Epilobium australe Poepp. et } \\
\text { Hausskn. ex Hausskn. }\end{array}$ & & & & & & & & & & & & 14,0 & & & & \\
\hline Gamochaeta spiciformis (Sch. Bip.) Cabrera & & & & & 29,0 & 12,5 & & & 13,3 & 62,5 & 58,0 & 29,0 & & & & \\
\hline Gaultheria pumila (L.f.) D.J. Middleton & 52,0 & & 77,0 & 19,0 & 22,5 & & 4,0 & & & & 19,0 & & 15,9 & 8,0 & 37,5 & \\
\hline $\begin{array}{l}\text { Gentianella magellanica (Gaudich.) } \\
\text { Fabris ex D.M. Moore }\end{array}$ & 1,0 & & 3,0 & & & & 13,0 & & 2,0 & 33,0 & & 2,0 & & 8,0 & & \\
\hline Gunnera magellanica Lam. & & & 44,0 & & 11,0 & & & & 11,3 & 8,0 & 13,2 & 92,0 & 12,7 & 17,0 & 89,8 & \\
\hline Hamadryas delfinii Phil. ex Reiche & & & & & & & & & & & & & & & 31,5 & \\
\hline Lagenophora hariotii Franchet & & & & & & & & & 8,0 & & & & & & & \\
\hline Leptinella scariosa Cass. & 6,0 & & 3,0 & & & & & & & & & & & & & \\
\hline Luzula alopecurus Desv. & 18,7 & & 1,0 & & 1,0 & & 65,0 & & 23,0 & & & & 1,0 & 12,5 & 49,3 & \\
\hline Lycopodium magellanicum (P. Beauv.) Sw. & & & 8,0 & & & & & & & & & & & & & \\
\hline Macrachaenium gracile Hook. f. & & & & & & & & & 8,0 & & & & & & & \\
\hline Marsippospermum grandiflorum (L. f.) Hook. & 14,1 & & 59,5 & & & & & & & & 15,0 & & 18,1 & & 15,2 & \\
\hline Nothofagus betuloides (Mirb.) Oerst. & & & & & & & & & 11,7 & 25,0 & & & & & & \\
\hline $\begin{array}{l}\text { Nothofagus pumilio (Poepp. et Endl.) Krasser } \\
\text { Osmorhiza berteroi DC. }\end{array}$ & & & 87,5 & 13,0 & 32,9 & 3,0 & 19,7 & 36,0 & 86,7 & 55,0 & 17,7 & 21,5 & & & & \\
\hline Perezia magellanica (L.f.) Less. & & & & & & & 2,0 & & & & & & & & & \\
\hline Phleum alpinum L. & 1,0 & & 87,5 & & 41,7 & & 63,3 & & 1,0 & & 77,5 & 25,0 & 92,0 & 33,0 & 11,2 & 26,3 \\
\hline Poa alopecurus (Gaudich.) Kunth & 9,0 & & 86,3 & 56,0 & 1,0 & & 13,3 & 33,5 & 5,0 & 22,5 & 7,0 & 18,0 & 37,5 & & 55,5 & \\
\hline Ranunculus minutiflorus Bertero ex Phil. & & & 14,5 & & & & & & & & & & & & & \\
\hline Ranunculus repens $\mathrm{L}$. & & & & & & & & & & & 31,0 & & & & & \\
\hline Ribes magellanicum Poir. & & & 44,0 & & & & 1,0 & & 25,0 & & 28,5 & 17,0 & & & & \\
\hline Rubus geoides Sm. & 4,0 & & 6,0 & & 17,2 & & 67,0 & & 75,0 & & 19,0 & & 25,0 & & 17,0 & \\
\hline Rumex acetosella L. & 36,7 & 17,0 & 79,5 & 7,0 & 1,0 & & & 12,0 & 7,0 & 12,5 & & & & & & \\
\hline Schizeilema ranunculus (D'Urv.) Domin & 13,0 & & 6,0 & & 19,0 & & 3,0 & & 4,0 & 8,0 & 75,0 & & & & & \\
\hline Senecio cf. patagonicus & & & & & & & & 8,0 & 3,0 & 15,0 & 82,0 & 13,0 & & & & \\
\hline $\begin{array}{l}\text { Senecio acanthifolius Hombr. \& } \\
\text { Jacquinot ssp. acanthifolius }\end{array}$ & & & & & 15,8 & & & & 15,8 & 8,0 & 8,0 & & & & & \\
\hline Taraxacum officinale Weber ex F.H. Wigg & & & 14,5 & 6,0 & & & 23,3 & 27,5 & 2,0 & & & & & & 8,0 & \\
\hline Trifolium repens $\mathrm{L}$. & 36,0 & & & & & & & & & & & & & & & \\
\hline Trisetum spicatum (L.) K.Richt. & 11,3 & & 18,7 & & 1,0 & 17,0 & 14,0 & & 19,4 & 17,0 & 46,0 & & & & 52,5 & \\
\hline Sphagnum magellanicum Brid.* & & 13,0 & 17,8 & & 52,0 & & 4,0 & & 23,8 & 25,0 & 13,0 & 1,0 & 14,0 & & 11,0 & 33,0 \\
\hline Líquenes* & & & 6,0 & & & & & & 6,0 & & 17,0 & & 25,0 & & & \\
\hline Riqueza de Plantas Vasculares & 17 & 2 & 25 & 9 & 19 & 4 & 18 & 7 & 24 & 12 & 19 & 11 & 15 & 5 & 18 & 2 \\
\hline
\end{tabular}




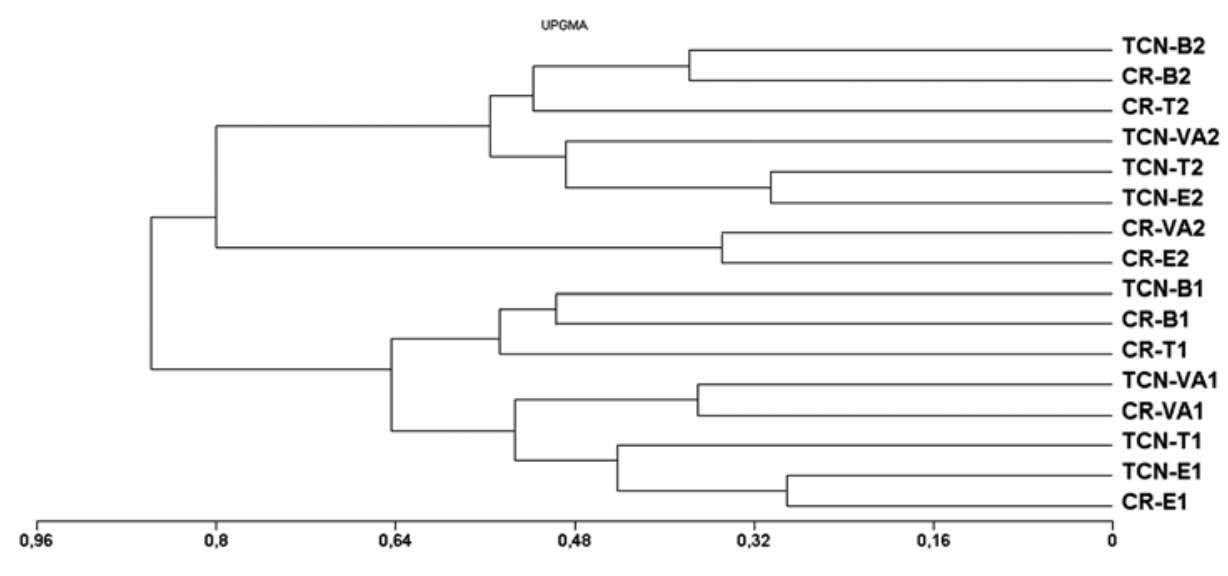

Fig. 2. Dendrograma de similitud basado en la cobertura de plantas vasculares entre los sitios de muestreo en Parque Karukinka, Tierra del Fuego. Índice de distancia de Bray-Curtis, método de agrupamiento UPGMA.

cuyas especies características son Poa alopecurus (Gaudich. ex Mirb.) Kunth, A.magellanica y Rumex acetosella L. Finalmente el clado D o cuarto grupo, conformado únicamente por parcelas del área degradada, reuniendo parcelas de los ecosistemas de bosque, estepa, ecotono y estepa andina; este clado está conformado por las especies D.flexuosa, P. alopecurus, Gunnera magellanica y Gentianella magellanica (Gaudich.) Fabris ex D.M. Moore.

El análisis de componentes principales (PCA) realizado sobre la estructura horizontal de la vege- tación (Fig. 3), muestra la segregación del grupo de parcelas correspondientes a las área degradada, las que se ubican cerca del origen de los ejes cartesianos, mostrando gran homogeneidad entre estas parcela con respecto a las del área de referencia. Las parcelas del área degradada presentan baja riqueza y baja cobertura de especies; en tanto que las parcelas del área de referencia se dispersan a lo largo del segundo eje de ordenación, donde las parcelas de estepa y estepa andina se separan notablemente de las parcelas de bosque y ecotono.

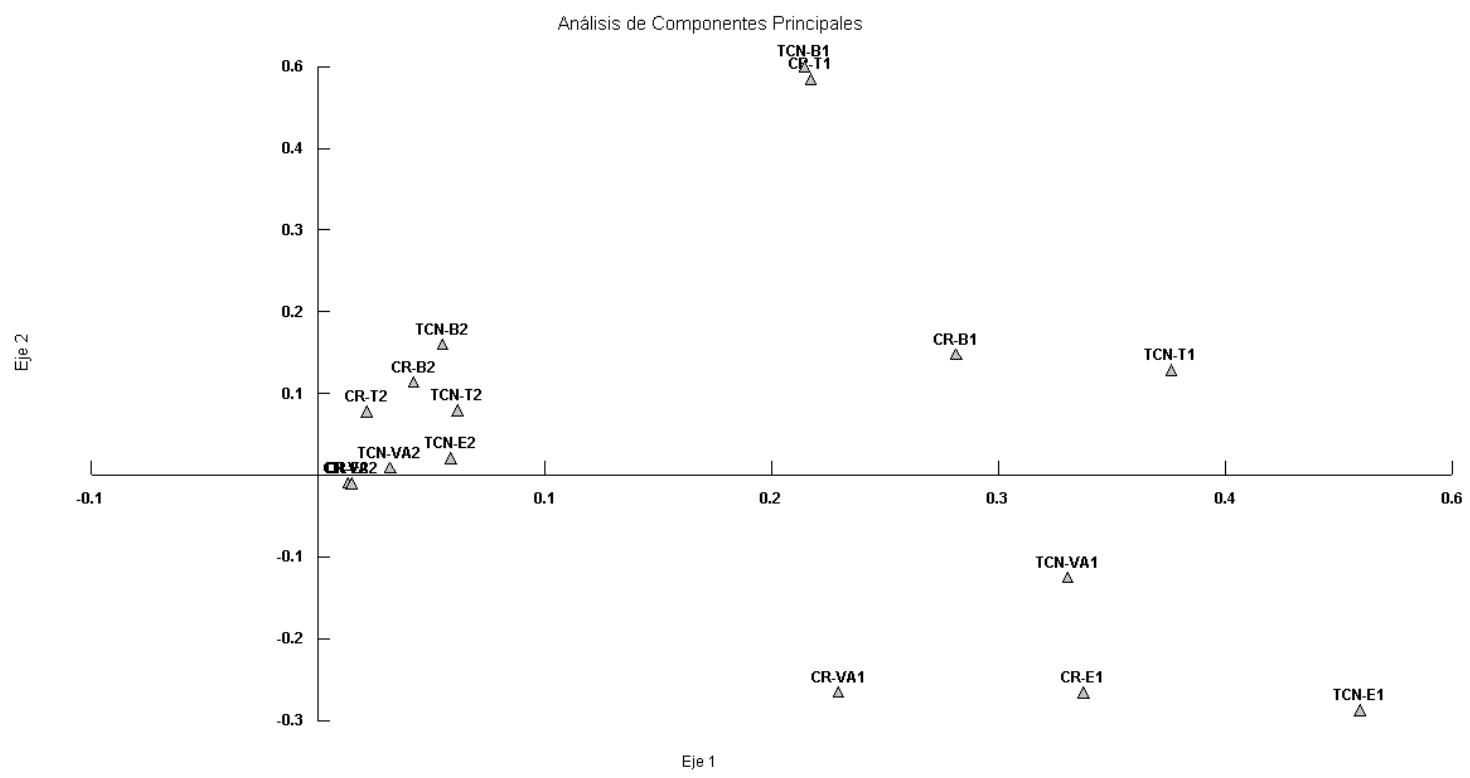

Fig. 3. Dispersión de los sitios de muestreo sobre los dos primeros ejes del Análisis de Componentes Principales. 


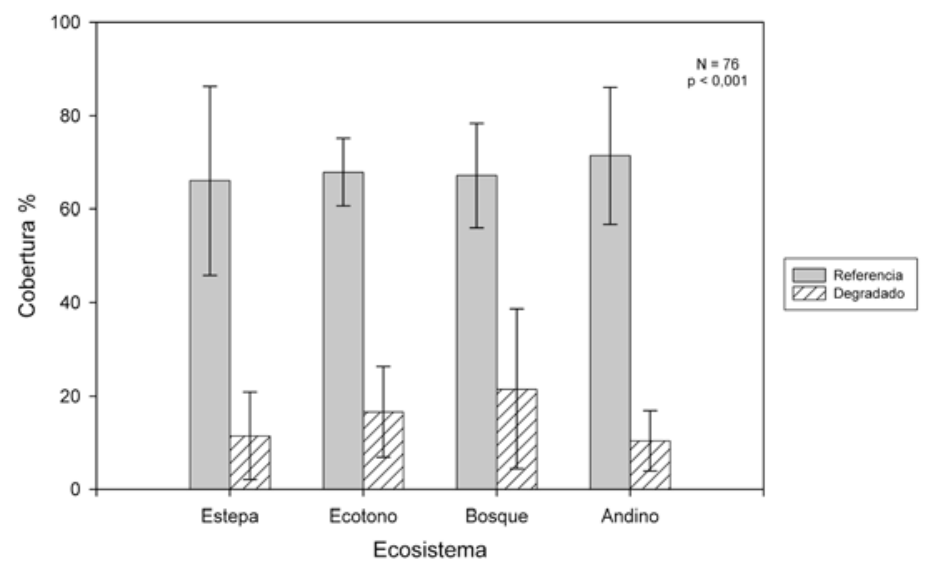

Fig. 4. Cobertura de especies en sitios de referencia y sitios degradados en los cuatro ecosistemas analizados en Parque Karukinka, Tierra del Fuego.

La dispersión de los sitios de muestreo a lo largo del eje de ordenación 2 estaría determinada por un gradiente de riqueza y cobertura.

Por su parte, los datos de cobertura de los sitios de referencia presentan diferencias significativas respecto de los sitios de área degradada en los cuatro ecosistemas estudiados (ANOVA $\mathrm{p}<0,05$; Tukey $\mathrm{p}<0,05$, Fig. 4). Los sitios de referencia presentan coberturas hasta 20 veces mayor que en sitios de área degradada. Estas diferencias confirman que el principal impacto de la construcción de caminos al formar taludes es la pérdida de cubierta vegetal. Por lo demás, no se hallaron diferencias significativas entre parcelas dentro de los sitios de área degradada, ni entre parcelas de área de referencia de diferentes ecosistemas (Tukey $\mathrm{p}<0,05$ ).
En cuanto a la riqueza de especies, esta presenta diferencias significativas (U Mann-Whitney $p<0,05)$ entre el número de especies observadas en los sitios de referencia respecto de los sitios degradados, en todos los ecosistemas. En la Figura 5, se observa gráficamente la similitud que hay entre la riqueza de especies en sitios degradados, y el número de especies compartidas entre ambos sitios, lo que sugiere que las especies que colonizan estas áreas degradadas provendrían mayormente desde el ecosistema de referencia.

Las especies más repetidas en sitios de área degradada son Acaena magellanica (16), Nothofagus pumilio (10) y Deschampsia flexuosa encontrada en 9 de los 38 taludes estudiados. Estas mismas especies, es decir A.magellanica, N.pumilio y D.flexuosa

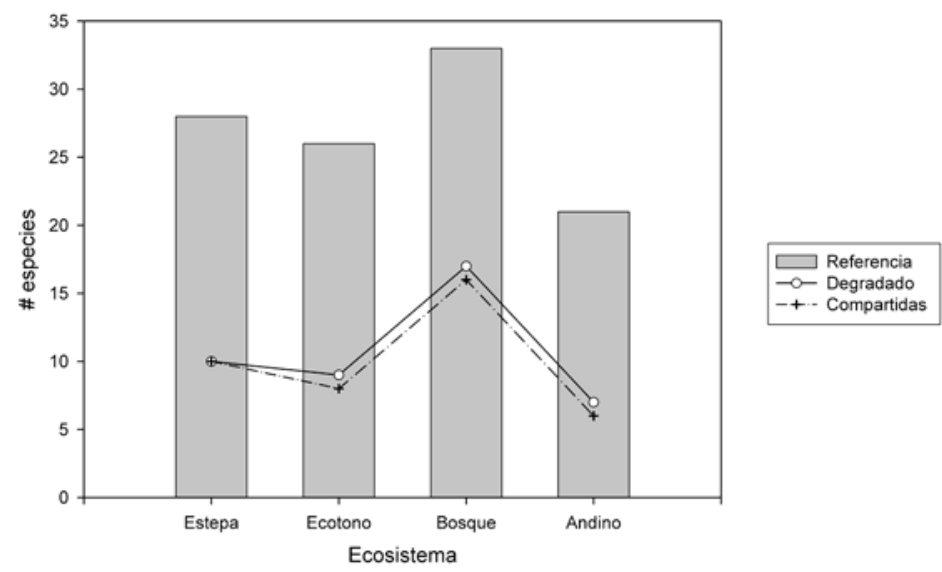

Fig. 5. Riqueza de especies en sitios de referencia y sitios degradados, y número de especies compartidas entre ambos sitios. 


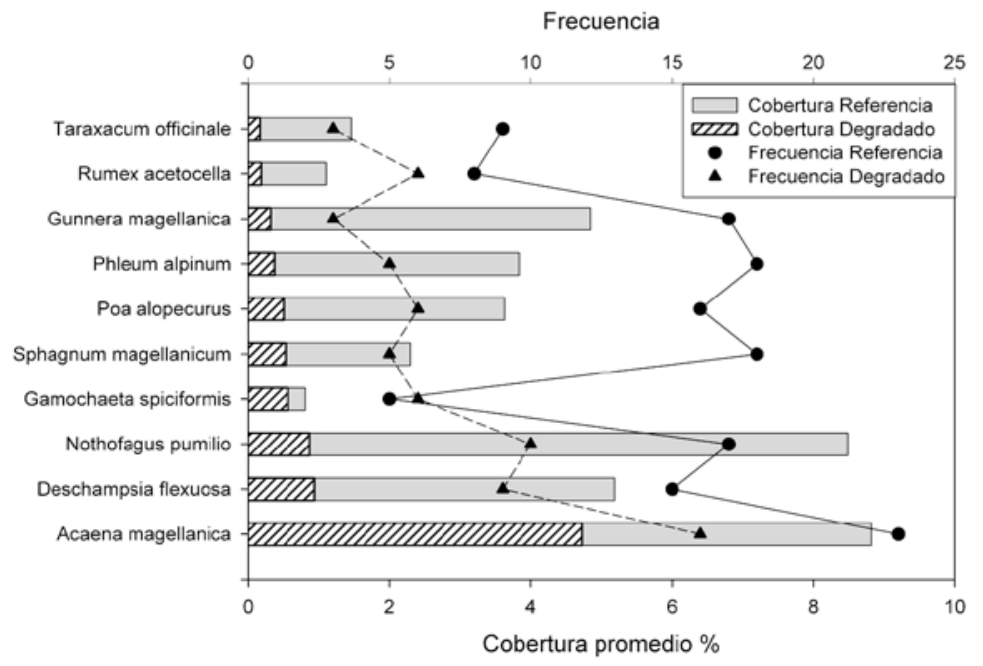

Fig. 6. Especies representativas de las áreas de referencia y taludes degradados de la Ruta Y-85, Parque Karukinka-Tierra del Fuego.

son aquellas con mayor porcentaje de cobertura en los sitios de área degradada. Mientras que en los sitios de referencia las especies que exhibieron mayor porcentaje de cobertura son nuevamente $A$. magellanica y N. pumilio, pero además Gunnera magellanica. En cuanto a las especies con mayor frecuencia en los sitios de referencia encontramos A.magellanica (23), Phleum alpinum (18), el musgo Sphagnum magellanicum (18), N.pumilio (17) y
Gunnera magellanica encontrada en 17 de los 38 sitios de referencia.

Análisis del patrón de colonización en sitios de área degradada

En cuanto a los sitios analizados que corresponden a áreas degradadas, se observa en el Análisis de Componentes Principales (Fig. 7) la

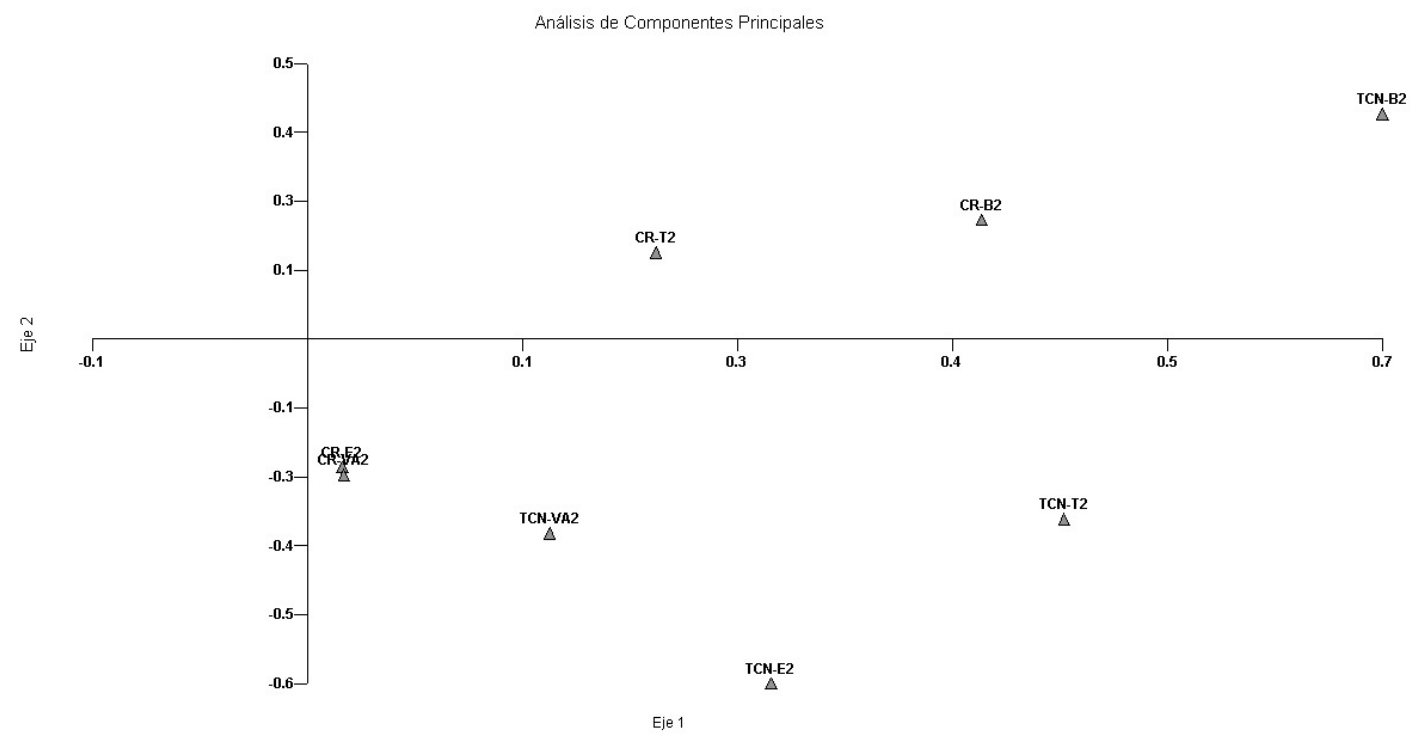

Fig. 7. Análisis de componentes principales (PCA) de los taludes degradados del Parque Karukinka realizado a partir de los datos de riqueza y cobertura de especies. 


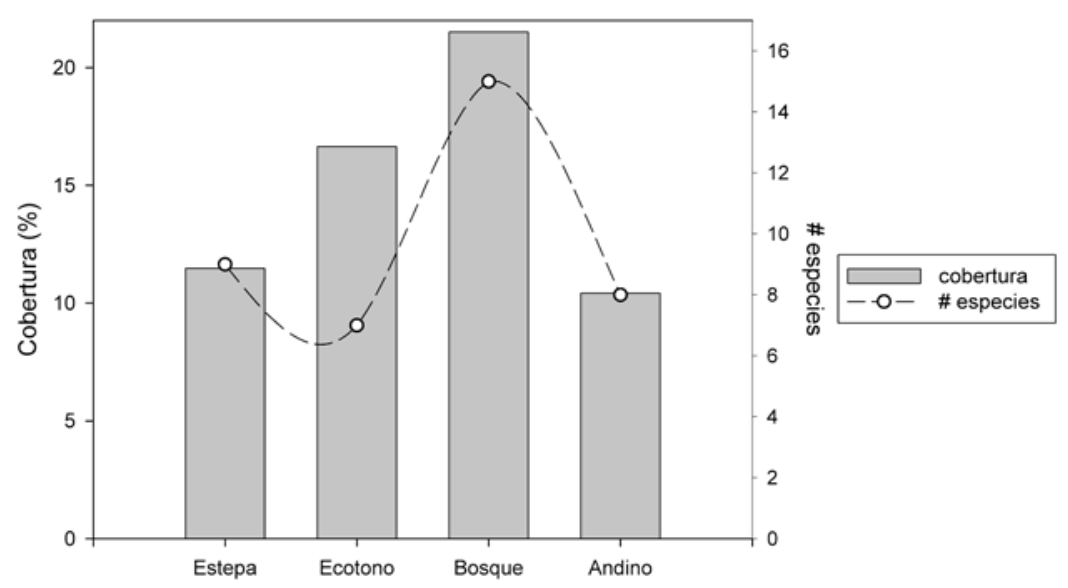

Fig. 8. Cobertura y riqueza de especies de sitios en áreas degradadas analizadas en Parque Karukinka, Tierra del Fuego- Chile.

dispersión de las parcelas según diferencias en la estructura horizontal de la vegetación. La dispersión de los puntos que representan las parcelas de áreas degradadas en torno al eje de ordenación 1 , puede ser explicado por las diferencias en riqueza y cobertura existentes entre las parcelas evaluadas en bosque versus las evaluadas en estepa y estepa andina, donde las primeras presentan alta riqueza y alta cobertura respecto a las segundas. No se observa un patrón de dispersión de las áreas degradadas relativo al tipo de sustrato observado en el corte ( $\mathrm{CR} / \mathrm{TCN})$, sino más bien como una función de la estructura del ecosistema de referencia.

La cobertura y riqueza de especies en área degradada en el ecosistema de bosque es mayor que en los demás ecosistemas (Fig. 8). En tanto que en la cobertura, le siguen el ecotono, estepa y finalmente la estepa andina, y en cuanto a la riqueza le siguen estepa, estepa andina y ecotono, probablemente ocurra este efecto debido a la mayor complejidad estructural del bosque respecto de los otros tres ecosistemas.

Por otra parte, ni cobertura ni riqueza de especies presentan un incremento con el tiempo (Fig. 8), contrario a lo que podría esperarse: que las áreas que fueron intervenidas primero (más antiguas) presenten mayor riqueza y cobertura de especies que aquellas intervenidas recientemente. Esto no se cumple, y se observa una respuesta basada en un factor estructural del hábitat circundante, considerando que el ecosistema de bosque presenta mayor complejidad de estratos verticales que sus ecosistemas vecinos, más que una respuesta al tiempo ocurrido desde la construcción del camino.

\section{DISCUSIÓN}

Con base en los resultados obtenidos en este estudio, se puede inferir que las áreas que han sido degradadas por efecto de la construcción del camino en el Parque Karukinka no han sido capaces de recuperarse por sí solas, ya que 14 años después del inicio de la construcción aún se observa el impacto de pérdida de cubierta vegetal en los taludes del camino, siendo del 89\% en taludes TCN y 63\% en taludes CR en ecosistema de bosque (Repetto, 2009). Porcentajes elevados, aún cuando según el presente estudio los sitios de área degradada en ecosistema de bosque presentan mayor cobertura y riqueza de especies en comparación con el resto de los ecosistemas, lo que podría deberse a la mayor complejidad de estratos que presenta el bosque, mayor lluvia de semillas, o las condiciones ambientales que pueda generar el ecosistema circundante.

En áreas donde las condiciones ambientales son favorables, la sucesión natural puede ser la mejor opción para una restauración "pasiva" (Bradshaw, 1997), pero cuando el ecosistema no es capaz de recuperarse, es necesario iniciar procesos de restauración "activa". En este sentido, es necesario identificar aquellas especies más susceptibles de colonizar exitosamente, adaptadas a las condiciones, y que provean de recursos y condiciones para la llegada de otras especies y que idealmente provengan del 
ecosistema de referencia (Cortina et al. 2004). En este estudio, dentro de las especies que colonizan los sitios de área degradada, y que son compartidas por el ecosistema de referencia, destacan: Acaena magellanica, Deschampsia flexuosa, Gunnera magellanica, Nothofagus pumilio y Phleum alpinum. En sitios de área degrada se observa una simplificación del ensamble vegetal que los cubre, en comparación a los sitios de referencia. Esta simplificación ocurre en desmedro de la vegetación arbustiva y de árboles del sitio (Repetto, 2009), por lo cual es importante evaluar el éxito de colonización de especies arbustivas en conjunto con especies rastreras como Acaena magellanica y Gunnera magellanica. Las especies arbóreas no son recomendables para revegetar taludes en este tipo de ecosistemas dominados por fuertes vientos, ya que pueden incrementar los riesgos de desprendimiento y caída en el camino.

Aún cuando no se observa un patrón de dispersión entre sitios de área degradada en CR y TCN, es importante considerar que tan solo los taludes de TCN podrían ser revegetados una vez que se realicen trabajos de estabilización (Repetto, 2009).

Este estudio cumple con el objetivo de evaluar la regeneración de la vegetación en taludes de caminos, identificando las especies que han sido capaces de colonizar estas áreas degradadas comparándolas con aquellas que se encuentran naturalmente en los sitios de referencia, lo que ha sido un primer paso para la selección de especies que requiere una revegetación del lugar. Es importante destacar la lenta o nula recuperación de las áreas degradadas que se observó en este estudio, lo que nos obliga a plantear la restauración activa de estas áreas, a través de una revegetación con especies nativas, como una opción para recuperar y estabilizar los taludes de la Ruta Y-85.

\section{AGRADECIMIENTOS}

Al Dr. Juan Marcos Henríquez por apoyarnos con este artículo desde sus inicios y al Dr. Julio Salcedo por facilitarnos su final. A Macarena Isla y Diego López, importantes socios en la extenuante toma de datos a lo largo de la Ruta Y-85. A los guardaparques de Karukinka por todo el apoyo brindado en terreno, en especial al equipo de rescate (Juan Sotomayor \& Claudio Moraga). A la Dirección de
Vialidad-Magallanes, y al Cuerpo Militar del Trabajo (CMT) por el constante interés en nuestro trabajo, y en mejorar la forma de construir caminos en nuestra región.

\section{LITERATURA CITADA}

Andrés, P. \& M. Jorba 2000. Mitigation strategies in some motorway embankments (Catalonia, Spain). Restoration Ecology, 8: 268-275.

Bradshaw, A.D. 1997. Restoration Ecology and Sustainable Development, Urbanska, K. \& N. R. Webb (eds.). Cambridge Univ. Press, Cambridge.

Bonham, C.H. 1989. Measurements for Terrestrial Vegetation. John Wiley \& Sons. New York. 338 pp.

Cortina, J., J. Bellot, A. Vilagrosa, R.N. Caturla, F.T. Maestre, E. Rubio, J.M. Ortiz de Urbina \& A. Bonet 2004. Restauración en Semiárido. En Vallejo V.R. \& J.A. Alloza (eds.) Avances en el estudio de la gestión del monte Mediterráneo. Fundación Centro de Estudios Ambientales del Mediterráneo (CEAM), Valencia. 345-406 pp.

Forman, R.T., D. Sperling, J.H. Bissonette, A.P. Clevenger, C.D. Cutshall, V.H. Dale, L. Fahrig, R. France, C.R. Goldman, K. Heanue, J.A. Jones, F.J. Swanson, T. Turrentine \& T.C. Winter 2003. Road Ecology: Science and Solutions. Island Press, Washington, D.C.

Foster, B. \& D. Tilman 2003. Seed limitation and the regulation of community structure in oak savanna grassland. Journal of Ecology, 91: 999-1007.

Gómez-Orea, D. \& M. Gómez-Villarino 2007. Consultoría e Ingeniería Ambiental. Editorial Mundi-Prensa. Madrid, España. 696 pp.

Jackson, S.T. \& R.J. Hobbs 2009. Ecological Restoration in the Light of Ecological History. Science, 325 (5940): 567-569.

National Research Council of the National Academies 2005. Assessing and Managing the Ecological Impacts of Paved Roads. The National Academies Press. 294 pp.

Nicolau, J.M. 2002. Runoff generation and routing on artificial slopes in a Mediterranean-continental environment: the Teruel coalfield, Spain. Hydrological Processes, 16: 631-647. 
Novák, J. \& K. Prach 2003. Vegetation succession in basalt quarries: pattern on a landscape scale. Applied Vegetation Science, 6: 111-116.

Repetto, F. 2009. Abriendo caminos para la conservación: restauración ecológica y desarrollo social en caminos públicos dentro de áreas protegidas. Proyecto fin de máster. Máster Oficial en Restauración de Ecosistemas, Universidad Complutense de Madrid, U. Politécnica de Madrid, U. de Alcalá y U. Rey Juan Carlos, España. 84 pp.

Saavedra, B., J.A. Simonetti \& K.H. Redford 2011. Chapter 14: Private Conservation, the example that the Wildlife Conservation Society builds from Tierra del Fuego. En: Figueroa EB (ed.) Biodiversity Conservation in the Americas: Lessons and Policy Recommendations, pp 357-391.

Snelder, D.J. \& R.B. Bryan 1995. The use of rainfall simulation tests to assess the influence of vegetation density on soil loss on degraded rangelands in the Baringo District, Kenya. Catena, 25 (1-4): 105-116.

Sokal, R.R. \& F.J. Rohlf 1995. Biometry: The Principles and practice of biological research. 3rd Edition. W.H. Freeman, New York. 887 pp.

Tormo, J., E. Bochet \& P. García-Fayos 2006. Is seed availability enough to ensure colonization success? An experimental study in road embankments. Ecological Engineering, 26: 224-230.

Vásquez-Barquero, A. 2009. Desarrollo local, una estrategia para tiempos de crisis. Conceptos Críticos. Universitas Forum 1 (2): 1-11.

Zamora, R., P. García-Fayos \& L. Gómez-Aparicio 2004. Las interacciones planta-planta y planta-animal en el contexto de la sucesión ecológica. En Valladares, F. (ed.) Ecología del bosque mediterráneo en un mundo cambiante. Ministerio de Medio Ambiente. EGRAF, Madrid. pp. 371-393. 
